\title{
Preface: Focus on the Main Research Interests of Professor Natale D'Alessandro
}

This Special Issue is in Memory of Professor Natale D'Alessandro, full Professor of Pharmacology at the University of Palermo, Italy, who made original contributions in various aspects of the clinical and preclinical pharmacology of cancer.

The first review is a contribution by a dear friend and colleague Benjamin Bonavida illustrating the professional scientific career of Natale D'Alessandro, in particular his contributions and investigations that have yielded significant results in a challenging field in cancer, dealing with cancer resistance to chemotherapeutic drugs, their underlying mechanisms, and means to reverse resistance.

In this context, the review of Cusimano et al. describes the research topic on a particularly aggressive and poorly responsive tumor, hepatocellular carcinoma (HCC), which is a very peculiar cancer because it presents several molecular alterations linked to the activation of survival and antiapoptotic signal pathways that are proteins in form and not easily targetable by even the newest targeted therapies. The aim of this review was to summarize studies on different molecules involved in the development, progression, and chemoresistance of HCC. In particular, they analyzed the role of inflammatory mediators, such as cyclooxygenase enzymes, nuclear factor $\kappa \mathrm{B}(\mathrm{NF}-\kappa \mathrm{B})$, interleukin 6 , as well as other important factors, such as Yin Yang 1 (YY1), in HCC. The mechanisms of resistance are multifactorial; between 1998 and 2016, Professor D'Alessandro and his co-workers focused their studies on three cellular models of innate and acquired resistance, acute myeloid leukemia, hepatocellular carcinoma, and triple negative breast cancer, which are all characterized by an involvement of the NF- $\mathrm{BB}$ pathway, reported by Poma et al. In the resistant phenotype, more strictly pharmacological factors are certainly involved, such as the P-glycoprotein, and biological factors such as apoptosis inhibitory proteins and Raf-1 kinase inhibitor protein, an important tumor suppressor and metastasis inhibitor, which enhances drug-induced apoptosis of cancer cells and YY1, a transcription factor involved in drug resistance. Particular attention has been paid to the multitarget molecules that can be considered as privileged compounds capable of attacking and reversing the resistant phenotype.

The review by Filippelli and co-workers shows the molecular mechanisms of resistance to antiangiogenic drugs. While the problems of resistance with conventional cytotoxic therapy are well known, the resistance to antiangiogenic drugs is new and not expected because of the low pattern of proliferation displayed by endothelial cells, which are the target of these drugs. With the approval of target therapy against tumor angiogenesis and in particular against the vascular endothelial growth factor (VEGF)/VEGF receptor system, researchers thought that resistance would no longer be a problem. However, beside the efficacy demonstrated by antiangiogenic drugs, resistance during prolonged drug treatments appears as a limiting feature. The review evidences the preclinical and clinical reports on the mechanisms of resistance to antiangiogenic drugs reported so far. Indeed, various mechanisms of resistance to antiangiogenic therapeutics have been discovered, either innate and depending on the host, or acquired by the tumor cells, especially as a consequence of induced hypoxia by antiangiogenic drugs and the redundancy of proangiogenic factors in the tumor microenvironment, and other forms of tumor neovascularization than sprouting angiogenesis. From a perspective point of view, knowledge of the mechanisms underneath antiangiogenic drug resistance could be of help in the choice of a more appropriate drug, the development of novel therapeutic strategies, the design of proper drug combination protocols, or new formulations of antiangiogenic strategies.

The review by Umezawa and co-workers focuses on the recent role of cancer-associated fibroblasts that are considered to activate cancer growth. Conophylline was isolated from the leaves of Ervatamia microphylla collected in Thailand. 
Conophylline was found to suppress secretions of various inflammatory cytokines by pancreatic cancer-associated fibroblasts. Moreover, it inhibited the growth of pancreatic cancer growth in mice together with gemcitabine. Conophylline is orally active. K-ras is one of the most important oncogenes in human oncogenesis. K-ras transfection to normal rat fibroblasts induces phenotypic change from flat morphology to round-shape morphology. They then screened compounds inducing flat morphology in K-Ras transformed fibroblasts from microbial culture filtrates and plant extracts. Conophylline induced flat morphology and inhibited cellular invasion in K-Ras-transformed normal rat kidney (K-Ras-NRK) cells. It also inhibited the growth of K-Ras-NRK tumor in mice. Thus, plant-derived alkaloid conophylline inhibited cancer growth directly and indirectly, and it may be a candidate as a new anticancer agent. The paper by Bergamo and Sava also helps to elucidate that many factors can influence the outcome of in vitro tests of cytotoxicity and suggests caution to speculate on possible therapeutic properties based on the results of simple and reductive in vitro tests of cytotoxicity. The expansion of metal-based complexes in the last 20 years has been very intense and many metals have been involved. Among the many compounds studied, the ruthenium-based complex NAMI-A embodies the unique paradigm of the ability to selectively inhibit and prevent the development and the growth of distant metastases originating from solid tumors in all the tumor models on which it has been tested. This activity can be detected only in vivo since the compound is virtually free of measurable direct cell cytotoxicity in vitro. Recently, a published paper reported on a significant in vitro cytotoxicity against some leukemic cells. In this study, the authors reproduce those experiments to further support this novel antileukemic activity that would have put NAMI-A on a new trajectory for development. Their results do not confirm the efficacy of NAMI-A in vitro against the human HL-60 promyelocytic leukemia cell line, supporting the lack of in vitro direct cell cytotoxicity of NAMI-A.

Guest Editors:

Monica Notarbartolo and Marina Ziche 